\begin{tabular}{l} 
O P E R A T I O N S R E S E A R C H A N D D E C I S I O N S \\
\hline No. 1
\end{tabular}

DOI: $10.37190 /$ ord200104

\title{
APPLICATION OF FUZZY PROGRAMMING TECHNIQUES TO SOLVE SOLID TRANSPORTATION PROBLEM WITH ADDITIONAL CONSTRAINTS
}

\author{
SHARMISTHA HALDER (JANA $)^{1}$, BISWAPATI JANA ${ }^{2 *}$ \\ ${ }^{1}$ Department of Mathematics, Midnapore College (Autonomous), Midnapore, West Bengal-721101, India \\ ${ }^{2}$ Department of Computer Science, Vidyasagar University, Midnapore, West Bengal-721102, India
}

An innovative, real-life solid transportation problem is explained in a non-linear form. As in real life, the total transportation cost depends on the procurement process or type of the items and the distance of transportation. Besides, an impurity constraint is considered here. The proposed model is formed with fuzzy imprecise nature. Such an interesting model is optimised through two different fuzzy programming techniques and fractional programming methods, using LINGO-14.0 tools followed by the generalized gradient method. Finally, the model is discussed concerning these two different methods.

Keywords: non-linear solid transportation problem, impurity constraints, fuzzy programming technique

\section{Introduction}

Hitchcock [12] in 1941 first developed the transportation problem, which is a subclass of linear programming where the constraints are equality or inequality. In the classical form, the problem minimises the total cost of transporting a product which is available at some sources and required in a various destination. Haley [31] in 1962 first developed the concept of STP/3D-transportation problem. The STP is the process of distributing certain products from its manufacturing points (sources) to the different demand points (destinations), using different conveyances and keeping in mind the factor of different transportation capacities and transportation costs, fixed charge costs, etc. so that total transportation cost is minimum. While dealing with real-life problems, vagueness appears in the transportation system due to insufficient information about the system or for some unforeseen problems as strikes, natural disasters, festivals, etc. As a result, consideration of the above uncertain environment in the transportation problem is important for practical purposes.

*Corresponding author, email address biswapatijana@gmail.com Received 18 May 2019, accepted 23 April 2020 
In real life, the transportation problem deals with non-linear, non-commensurable, multiple and conflicting objective functions. Researchers in the past calculated distance function to apply the mathematical model of the non-linear multi-objective transportation problem (NMOTP). In our proposed case the singular objective function of the transportation problem gets to be nonlinear. After Zadeh's [10] introduction to fuzzy set theory in 1965, Zimmermann [11] uses the fuzzy programming technique with some added membership functions to solve multi-objective LPP (linear programming problem). The result was efficient. Bit et al. again use the fuzzy programming technique to solve multi-objective transportation problems by selecting an appropriate linear membership function. In the year 1999, Biswal and Verma [3] apply a fuzzy programming technique to evaluate the optimal compromise solution of a non-linear multi-objective transportation problem. Jimenez and Verdegay [6] come up with a technique to solve a fuzzy solid transportation problem. Discussion about fuzzy programming and additive fuzzy programming for multi-objective transportation problems can be found in [1] and [2]. Lin and Wen [4] propose the method for solving the fuzzy assignment problem. Introduction to the fuzzy transportation problem with additional restriction is given by Dutta and Murthy [5]. Singh and Saxena [8] study the multi-objective time transportation problem with additional impurity restriction. Generally, more than one objective function is considered in a transportation problem. In real scenarios, it is practical to assume that the quantity of an item that can be sent on a particular path is bounded by the capacity of the path. Also, Appa [13] give us ideas about the different variables that can occur in a transportation problem. In this paper, the formulation of a numerical model of the nonlinear transportation problem is shown. In this non-linear STP, one part of the objective function is linear with fixed transportation cost and the second part is non-linear with unit transportation fuzzy cost is varied with distance from some source to the origin.

The structure of the paper is as follows. Section 1 is the introduction, and literature survey related to the paper is presented in Section 2. Some basic ideas about solid transportation problem are given in Section 3. In Section 4, the model definition and formulation are presented. In Section 5, solution techniques are briefly discussed. A numerical problem using real-life data is presented and solved in Section 6. In Section 7, sensitivity analysis are addressed. The conclusion and scope of further research are shown in the last Section 8.

\section{Literature survey}

The imprecise nature of the mathematical model is the trend of mathematical research of the last few decades. At an earlier age, the imprecise nature transformed into a crisp nature through different simple definition cum methods, like fuzzy centroid method, possibility necessity method, interval method, etc. But during the last few 
years, the fuzzy programming method marks a new era in the area of imprecise nature of the model. So, the study of the fuzzy programming technique in solid transportation problems is becoming much popular in recent years. Liang [21] describes a fuzzy linear programming technique in an interactive way. Verma et al. [22] propose a special type of non-linear membership function to solve the multi-objective TP and compare the obtained result with linear membership function. Li and Lai [23] represent a fuzzy programming technique where various objectives are synthetically considered with the marginal revolution. Kumar [20] suggests a simple method for solving type-2 and type-4 fuzzy transportation problem. In 2014, Kumar [18] examines a systematic approach for solving the mixed intuitionistic fuzzy transportation problem. In 2018, Kumar [20] compares his earlier method with the recently obtained by the software-based approach. Recently, Anukokila [14] offers a fuzzy goal programming method for solving multi-objective transportation problem, and Kumar [15] very carefully develops a PSK method for solving the mixed fuzzy solid transportation problem. Ramakrishna [25] describes a variation of Vogel's approximation method for finding an initial feasible solution to the TP. Shafaat and Goyal [26] introduce a procedure for ensuring an improved solution for a problem with a single degenerated basic feasible solution. Arsham and Khan [27] give an algorithm that is faster than simplex, more general than a stepping-stone, and simpler than both in solving the general transportation problems. Gass [28] describes various aspects of TP methodologies and computational results.

\section{Preliminaries. Solid (3D) transportation problem}

The solid transportation problem (STP) is a generalization of the well-known transportation problem (TP) in which three-dimensional properties are taken into account in the objective and constraint set instead of source and destination. The STP was first stated by Shell [24]. In many industrial problems, a homogeneous product is delivered from an origin to a destination, using different modes of transport called conveyances, such as trucks, cargo flights, goods trains, ships, etc. These conveyances are taken as the third dimension. A solid transportation problem can be converted to a classical transportation problem by considering only a single type of conveyance (Fig. 1).

We consider $m$ origins (or sources) $O_{i}(i=1,2, \ldots, m), n$ destinations (i.e., demands) $D_{j}(j=1,2, \ldots, n)$, and $K$ conveyances $E_{k}(k=1,2, \ldots, K) . K$ conveyances, i.e., the different modes of transport, may be trucks, cargo flights, goods trains, ships, etc. Let $a_{i}$ be the amount of a homogeneous product available at $i$ th origin, $b_{j}$ the demand at $j$ th destination and $e_{k}$ represent the amount of product which can be transported by the $k$ th conveyance. The $C_{i j k}$ is the unit transportation cost from $i$ th source to $j$ th destination, 
using the $k$ th conveyance. The variable $x_{i j k}$ represents the unknown quantity to be transported from origin $O_{i}$ to destination $D_{j}$ employing $k$ th conveyance. The mathematical form of STP is

$$
\min Z=\sum_{i=1}^{m} \sum_{j=1}^{n} \sum_{k=1}^{k} c_{i j k} x_{i j k}
$$

subject to

$$
\begin{gathered}
\sum_{j=1}^{n} \sum_{k=1}^{k} x_{i j k}=a_{i}, i=1,2,3, \ldots, m \\
\sum_{i=1}^{m} \sum_{k=1}^{k} x_{i j k}=b_{j}, \quad j=1,2,3, \ldots, n \\
\sum_{i=1}^{m} \sum_{j=1}^{n} x_{i j k}=c_{k}, \quad k=1,2,3, \ldots, k \\
x_{i j k} \geq 0, \quad \forall i, j, k
\end{gathered}
$$

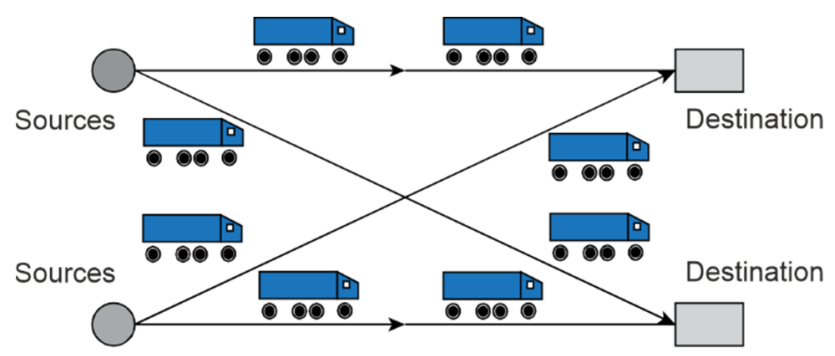

Fig. 1. Illustration of STP

\section{Model definition and formulation}

\subsection{Notations}

\section{Subscripts}

$i-$ index for source/origin for all $i=1,2, \ldots, m$

$j-$ index for destination for all $j=1,2, \ldots, n$ 


\section{Decision variables}

$w_{i j} \quad-$ units transported from $i$ th origin to $j$ th destination

$\left(x_{i}, y_{i}\right)$ - position of the $i$ th origin

$z_{1} \quad-$ total transportation cost from $i$ th origin to $j$ th destination

\section{Parameters}

$h_{i j} \quad-$ production cost per unit delivered from $i$ th origin to $j$ th destination

$c_{i j} \quad-$ transportation cost per unit delivered from warehouses $i$ to markets $j$

$a_{i} \quad$ - total available supply for each source (or origin)

$a_{i} \quad-$ total available supply for each source (or origin)

$b_{j} \quad-$ total demand of each destination $j\left(p_{j}, q_{j}\right)$ position of the $j$ th destination

$d_{i j} \quad-$ distance of per unit delivered from $i$ th warehouses to $j$ th markets

\subsection{Model formulation}

Let us consider a transportation problem with $m$ origins $O_{i}(i=1,2, \ldots, m)$ and $n$ destinations $\left.D_{j} \mathrm{C} j=1,2, \ldots, n\right)$ in which the position $\left(x_{i}, y_{i}\right)$ of origins is to be decided concerning the position of destination $\left(p_{j}, q_{j}\right)$ of the units of transportation $w_{i j}$ from $i$ th origin to $j$ th destination. Also to be decided: the first part of the objective function is the cost associated with the amount to be transported, and the second part is associated with the distance from the origin to destinations. Hence, the objective function of the non-linear solid transportation problem is as follows:

$$
z_{1}=\min \left\{\sum_{i=1}^{m} \sum_{j=1}^{n} \tilde{h}_{i j} w_{i j}+\sum_{i=1}^{m} \sum_{j=1}^{n} \tilde{c}_{i j} d_{i j} y_{i j}\right\}
$$

where

$$
y_{i j}=\left\{\begin{array}{l}
1, \text { if } w_{i j} \neq 0 \\
0, \text { if } w_{i j}=0
\end{array}\right.
$$

and

$$
d_{i j}=\sqrt{\left(x_{i}-p_{j}\right)^{2}+\left(y_{i}-q_{j}\right)^{2}}
$$

For the $i$ th origin $O_{i}$ to the total amount shipment $\sum_{j=1}^{n} w_{i j}$ cannot exceed its availability $a_{i}$. Hence, we must have the availability constraint 


$$
\sum_{j=1}^{n} w_{i j} \leq a_{i}, \quad i=1,2, \ldots, m
$$

In other words, the total incoming shipment at $j$ th destination is $\sum_{i=1}^{m} w_{i j}$, which should satisfy its requirement or demand. That is, we must support the demand constraint

$$
\sum_{i=1}^{m} w_{i j} \geq b_{j}, \quad j=1,2, \ldots, n
$$

In the transportation system, if an item damaged/broke during the time of transportation, then the demand point cannot receive $100 \%$ perfect item as supplied from the sources. For such type of item, the demand for perfect (undamaged/unbreakable) item by the demand point yields an impurity constraint with the help of impurity factor $f_{i}$ as in the form

$$
\sum_{i=1}^{m} f_{i} w_{i j} \leq g_{j}, \quad j=1,2, \ldots, n
$$

Non-negativity constraints on decision variables: $w_{i j} \geq 0, \forall i, j$.

\section{Solution procedure}

For a solid transportation problem, the objective is to find the decision variables which minimise the total cost of transportation. For an STP, every solution is a feasible solution, since it needs to satisfy the feasibility condition (cf. equations (1)-(5), Section 3 ). If the number of non-zero solutions is less than $(m+n+k-2)$, then such a solution is known as a degenerate solution which yields multiple basic feasible solutions. Among the basic feasible solutions, the optimum solution is to find out one based on the objective function (minimisation of total transportation cost).

\subsection{Method 1 proposed by Kanti Swarap}

Step 1. The transportation costs of many real-world applications are not deterministic numbers. Consider a manufacturing company which provides different products 
for different warehouses and transports to different destinations. In that case, the company usually restricts the transported $\cos t c_{i j}^{0}$ from $i$ th origin to $j$ th destinations, and the transported costs $c_{i j}$ vary from the distance where the product or goods can be shipped from $i$ th warehouses to $j$ th market. Here, we assume a minimum cost for the amount of product shipped from $i$ th origin to $j$ th destination.

In this case, the fuzzy costs $\tilde{c}_{i j}=\left(\alpha_{i j}, \beta_{i j}\right)$ are subnormal fuzzy numbers having strictly increasing linear membership functions, where $\alpha_{i j}$ is the least cost associated with the amount to be shipped from $i$ th origin to $j$ th destination and $\alpha_{i j}$ is the least cost associated with the amount to be shipped from $i$ th origin to $j$ th destination with the highest quality of the product. Without loss of generality, it is assumed that $\alpha_{i j}>\beta_{i j}>0$. The membership function of $\tilde{c}_{i j}$ (Fig. 2) as the strictly increasing linear function is

$$
\mu_{i j}\left(c_{i j}\right)=\left\{\begin{array}{l}
q_{i j} \text { if } c_{i j} \geq \beta_{i j} \\
q_{i j} \frac{c_{i j}-\alpha_{i j}}{\beta_{i j}-\alpha_{i j}} \text { if } \alpha_{i j} \leq c_{i j} \leq \beta_{i j} \\
0 \text { otherwise }
\end{array}\right.
$$

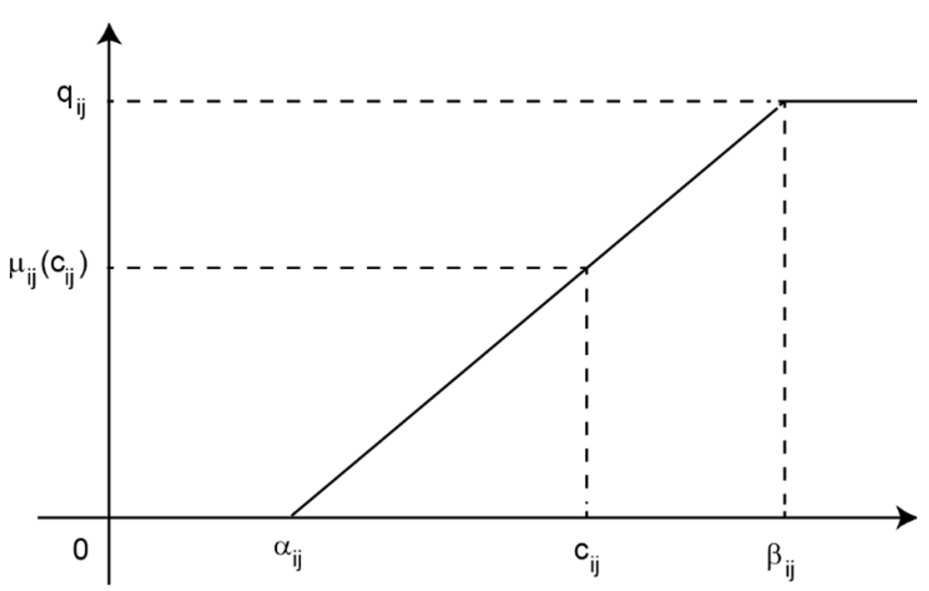

Fig. 2. Membership function of $\tilde{c}_{i j}$

The condition $w_{i j}>0$ is added to (10) because there is no real expense if $w_{i j}=0$ in any feasible solution $w=\left\{w_{i j}, 1 \leq i \leq m, 1 \leq j \leq n\right\}$ of (6). We use the notation $<\alpha_{i j}, \beta_{i j}>$ to denote $\tilde{c}_{i j}$. Matrix $\tilde{c}_{i j}$ is shown as follows: $\left[\tilde{c}_{i j}\right]=\left[<\alpha_{i j}, \beta_{i j}>\right]_{m \times n}$. The quality matrix $\left[q_{i j}\right]$ is

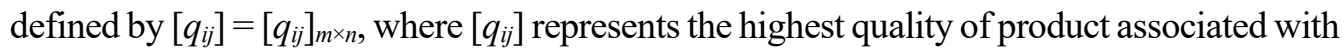
the amount transported from $i$ th warehouses to $j$ th market and $0<\left[q_{i j}\right] \leq 1$. 
Step 2. Let $\tilde{c}_{T}$ denote the total cost, and the numbers $a$ and $b$ are defined as the lower and upper bounds of the total cost, respectively. We define the membership function of $\tilde{c}_{T}$ as the linear monotonically decreasing function in (8), and use the notation $<a, b>$ to denote fuzzy interval $\tilde{c}_{T}$. Numbers $a$ and $b$ are constants and subjectively chosen by the manager. We may take $a$ as the minimum cost of the transportation problem with $\alpha_{i j}$ 's as costs and $b$ is the maximum cost of the transportation problem with $\beta_{i j}$ 's as costs, the demand and supply values in both being same as those of problem (6). The membership function of the total cost (Fig. 3) is

$$
\mu_{T}\left(c_{T}\right)=\mu_{T}\left(z_{1}\right)=\left\{\begin{array}{l}
1 \quad \text { if } \quad c_{T} \leq a \\
\frac{b-z_{1}}{b-a}=\frac{b-c_{T}}{b-a} \text { if } a \leq c_{T} \leq b \\
0 \quad \text { if } c_{T} \geq b
\end{array}\right.
$$

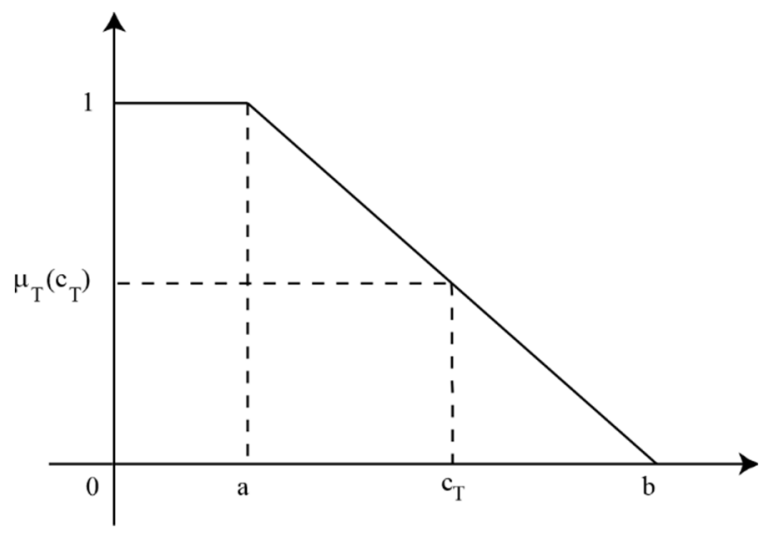

Fig. 3. Membership function of $\tilde{c}_{T}$

Step 3. We choose Bellman-Zadeh's criterion [9] which maximises the minimum of the membership functions corresponding to that solution, i.e.,

$$
\max \left\{\min \left(\mu_{i j},\left(\mu_{T}\left(c_{T}\right)\right)\right\}\right.
$$

where $w_{i j}$ is an element of a feasible solution $w=\left\{w_{i j} ; 1 \leq i \leq m, 1 \leq j \leq n\right\}$ of (6). Then, we can represent the problem as follows:

$$
\max \left\{\min \left(\mu_{i j},\left(\mu_{T}\left(c_{T}\right)\right) ; w_{i j}>0\right\}\right.
$$

subject to 


$$
\begin{aligned}
& \sum_{j=1}^{n} w_{i j} \leq a_{i}, \quad i=1,2, \ldots, m \\
& \sum_{i=1}^{m} w_{i j} \geq b_{j}, \quad j=1,2, \ldots, n \\
& \sum_{i=1}^{m} f_{i} w_{i j} \leq g_{j}, \quad j=1,2, \ldots, n
\end{aligned}
$$

Non-negativity constraints on decision variables: $w_{i j} \geq 0, \forall i, j$

Step 4. We further restrict the transportation cost to be less than or equal to $\beta_{i j}$ since any expense exceeding $\beta_{i j}$ is useless. By membership function of (10) and (11), we can further represent (14) as the following equivalent model.

Max $\lambda$ subject to

$$
\begin{aligned}
& \lambda \leq q_{i j} \frac{c_{i j}^{\lambda}-\alpha_{i j}}{\beta_{i j}-\alpha_{i j}} \\
& \lambda \leq \frac{b-z_{1}}{b-a} \\
& \sum_{j=1}^{n} w_{i j} \leq a_{i}, \quad i=1,2, \ldots, m \\
& \sum_{i=1}^{m} w_{i j} \geq b_{j}, \quad j=1,2, \ldots, n \\
& \sum_{i=1}^{m} f_{i} w_{i j} \leq g_{j}, \quad j=1,2, \ldots, n \\
& w_{i j} \geq 0, \quad \forall i, j \quad \text { for } i=1,2, \ldots, m, \quad j=1,2, \ldots, n \\
& c_{i j}^{\lambda} \leq \beta_{i j}, \quad \forall i, j, \quad 0 \leq \lambda \leq 1
\end{aligned}
$$

where $c_{i j}^{\lambda}$ denotes the $\alpha$-cut of $\tilde{c}_{i j}$. In (15), since $w_{i j}, c_{i j}^{\lambda}$ and $\lambda$ are all decision variables, it can be treated as a mixed integer nonlinear programming model. We first define the set $E=(i, j)$ as the set of all pairs $(i, j)$ where $w_{i j}$ is an element of the feasible solution $w=\left\{w_{i j}\right.$; $1 \leq i \leq m, 1 \leq j \leq n\}$ of (6) and confine our discussion based on $E$, then we can simplify (15) as follows:

$\operatorname{Max} \lambda$ subject to

$$
\lambda \leq q_{i j} \frac{c_{i j}^{\lambda}-\alpha_{i j}}{\beta_{i j}-\alpha_{i j}}, \quad(i, j) \in E
$$




$$
\begin{gathered}
\lambda \leq \frac{b-\Sigma_{(i, j)} c_{i j}^{0} w_{i j}-\Sigma_{(i, j)} c_{i j}^{\lambda} d_{i j} y_{i j}}{b-a} \\
c_{i j}^{\lambda} \leq \beta_{i j} \text { for }(i, j) \in E
\end{gathered}
$$

We let $h_{i j}=\beta_{i j}-c_{i j}^{\lambda} \geq 0$. Then, (16) and (17) can be expressed as follows:

$\operatorname{Max} \lambda$

$$
\begin{gathered}
\lambda \leq q_{i j} \frac{\beta_{i j}-h_{i j}-\alpha_{i j}}{\beta_{i j}-\alpha_{i j}} \text { for }(i, j) \in E \\
\lambda \leq \frac{b-\Sigma_{(i, j)} c_{i j}^{0} w_{i j}-\Sigma_{(i, j)}\left(\beta_{i j}-h_{i j}\right) d_{i j} y_{i j}}{b-a} \\
h_{i j}, \lambda, y_{i j} \geq 0 \text { for }(i, j) \in E
\end{gathered}
$$

Theorem 1. Let $\lambda \mathrm{w}$ be the optimal value of (18)-(21). Suppose

$$
b<\frac{\sum_{i, j} c_{i j}^{0} w_{i j}-\Sigma_{i, j}\left(\beta_{i j}-h_{i j}\right) d_{i j} y_{i j}}{1-\min _{(i, j)}\left(q_{i j}\right)}
$$

then

$$
\begin{gathered}
\lambda_{w}=q_{i j} \frac{\beta_{i j}-h_{i j}-\alpha_{i j}}{\beta_{i j}-\alpha_{i j}} \text { for }(i, j) \in E \\
\lambda=\frac{b-\Sigma_{(i, j)} c_{i j}^{0} w_{i j}-\Sigma_{(i, j)}\left(\beta_{i j}-h_{i j}\right) d_{i j} y_{i j}}{b-a}
\end{gathered}
$$

Proof. The problem (18)-(21) can be written into a linear programming model as $\operatorname{Max} \lambda$ subject to

$$
\begin{gathered}
h_{i j}+\lambda \frac{\beta_{i j}-\alpha_{i j}}{q_{i j}} \leq\left(\beta_{i j}-\alpha_{i j}\right) \text { for }(i, j) \in E \\
-\Sigma_{(i, j)} h_{i j} d_{i j} y_{i j}+(b-a) \lambda \leq b-\Sigma_{(i, j)} c_{i j}^{0} w_{i j}-\Sigma_{(i, j)} \beta_{i j} d_{i j} y_{i j}
\end{gathered}
$$




$$
h_{i j}, \lambda \geq 0 \text { for }(i, j) \in E
$$

We obtain the dual problem of the above problem as

$$
\min \Sigma_{(i, j)}\left(\beta_{i j}-\alpha_{i j}\right) v_{i}+\left\{b-\Sigma_{(i, j)} c_{i j}^{0} w_{i j}-\Sigma_{(i, j)} \beta_{i j} h_{i j} y_{i j}\right\} v_{n+1}
$$

subject to

$$
\begin{gathered}
v_{i}-v_{n+1} \geq 0 \text { for }(i, j) \in E \\
\Sigma_{(i, j)} \frac{\beta_{i j}-\alpha_{i j}}{q_{i j}} v_{i}+(b-a) v_{n+1} \geq 1 \\
v_{i} \geq 0, v_{n+1} \geq 0 \text { for } i=1,2, \ldots, n
\end{gathered}
$$

Let $s_{1}, s_{2}, \ldots, s_{n+1}$ be the slack variables of (23) and (24), respectively. Similarly, let $u_{1}, u_{2}, \ldots, u_{n+1}$ be the surplus variable of (27) and (28), respectively. Since

$$
b<\frac{\Sigma_{i, j} c_{i j}^{0} w_{i j}-\Sigma_{i, j}\left(\beta_{i j}-h_{i j}\right) d_{i j} y_{i j}}{1-\min _{(i, j)}\left(q_{i j}\right)}
$$

we have

$$
\min _{(i, j)}\left(q_{i j}\right)>\frac{b-\Sigma_{i, j} c_{i j}^{0} w_{i j}-\Sigma_{i, j}\left(\beta_{i j}-h_{i j}\right) d_{i j} y_{i j}}{b-a}
$$

By (20), we have $\alpha \min _{(i, j)}\left(q_{i j}\right)$ and $\forall h_{i j}>0$. As based on the complementary slackness theorem, we obtain $u_{1}=u_{2}=\ldots=u_{n}=0$. Hence, $v_{i}-v_{n+1}=0$ for $i=1,2, \ldots, n$ and $v_{1}=v_{2}=\ldots=v_{n}=v_{n+1}$. If $v_{1}=v_{2}=\ldots=v_{n}=v_{n+1}=0$, there is a contradiction to (28). Therefore, we have $v_{1}=v_{2}=\ldots=v_{n}=v_{n+1}>0$, and, again, by the complementary slackness theorem, we find $s_{1}=s_{2}=\ldots=s_{n+1}=0$, the proof is then completed. In most of the real-life problems, the upper bound condition of the total $\operatorname{cost} c_{T}$, i.e,

$$
b<\frac{\sum_{i, j} c_{i j}^{0} w_{i j}-\Sigma_{i, j}\left(\beta_{i j}-h_{i j}\right) d_{i j} y_{i j}}{1-\min _{(i, j)}\left(q_{i j}\right)}
$$

can be just satisfied. Therefore, we concentrate our discussion on this situation. 
Theorem 2. Let $\lambda_{w}$ be the optimal value of (18)-(21), and

$$
b<\frac{\Sigma_{i, j} c_{i j}^{0} w_{i j}-\Sigma_{i, j}\left(\beta_{i j}-h_{i j}\right) d_{i j} y_{i j}}{1-\min _{(i, j)}\left(q_{i j}\right)}
$$

Also, let

$$
\gamma_{i j}=\frac{\left(\beta_{i j}-\alpha_{i j}\right)}{q_{i j}} \text { for } i=1,2, \ldots, m, j=1,2, \ldots, n
$$

then

$$
\lambda_{w}<\frac{b-\Sigma_{i, j} c_{i j}^{0} w_{i j}-\Sigma_{i, j} \alpha_{i j} d_{i j} y_{i j}}{b-a+\Sigma_{i, j} \gamma_{i j} d_{i j} y_{i j}}
$$

Proof. By Theorem 1, assuming the solution to be non-degenerate, we have

$$
\begin{aligned}
& \lambda_{w}=\frac{\left(\beta_{i j}-h_{i j}-\alpha_{i j}\right) d_{i j} y_{i j}}{\gamma_{i j} d_{i j} y_{i j}} \text { for }(i, j) \in E \\
& \lambda=\frac{b-\Sigma_{(i, j)} c_{i j}^{0} w_{i j}-\Sigma_{(i, j)}\left(\beta_{i j}-h_{i j}\right) d_{i j} y_{i j}}{b-a}
\end{aligned}
$$

Hence, after transformations we get

$$
\begin{aligned}
\lambda_{w} & =\frac{b-\Sigma_{(i, j)} c_{i j}^{0} w_{i j}-\Sigma_{(i, j)}\left(\beta_{i j}-h_{i j}\right) d_{i j} y_{i j}+\Sigma_{(i, j)}\left(\beta_{i j}-h_{i j}-\alpha_{i j}\right) d_{i j} y_{i j}}{\left(b-a+\Sigma_{i, j} \gamma_{i j} d_{i j} y_{i j}\right)} \\
& =\frac{b-\Sigma_{i, j} c_{i j}^{0} w_{i j}-\Sigma_{i, j} \alpha_{i j} d_{i j} y_{i j}}{b-a+\Sigma_{i, j} \gamma_{i j} d_{i j} y_{i j}}
\end{aligned}
$$

It should be noted that this method applies to problems where the objective function $f$ is any general function having continuous partial derivatives. Here it will be better to start with a basic feasible solution instead of any feasible solution to the problem, because of its optimization criteria. This method leads to less computational work. Also for this method, if the objective function is convex or quasi-convex, then the local optimum is global. 


\subsection{Method 2. Fractional programming model}

By Theorem 2 and (15), (29) can be restated as

$$
\max \left\{\frac{\left(b-\Sigma_{i, j} c_{i j}^{0} w_{i j}-\Sigma_{i, j} \alpha_{i j} d_{i j} y_{i j}\right)}{\left(b-a+\Sigma_{i, j} \gamma_{i j} d_{i j} y_{i j}\right)}\right\}
$$

subject to

$$
\begin{aligned}
& \sum_{j=1}^{n} w_{i j} \leq a_{i}, \quad i=1,2, \ldots, m \\
& \sum_{i=1}^{m} w_{i j} \geq b_{j}, \quad j=1,2, \ldots, n \\
& \sum_{i=1}^{m} f_{i} w_{i j} \leq g_{j}, \quad j=1,2, \ldots, n \\
& w_{i j} \geq 0, \quad \forall i, j, \quad i=1,2, \ldots, m, \quad j=1,2, \ldots, n
\end{aligned}
$$

This is a linear fractional programming problem and its optimal solution may be obtained by the Kanti Swarup algorithm [7]. Now, $h_{i j}$ for $(i, j) \in E$ can be obtained from

$$
\lambda_{w}=\frac{\beta_{i j}-h_{i j}-\alpha_{i j}}{\gamma_{i j}}
$$

Then, the fuzzy costs corresponding to the maximal value of $\lambda$ are given by $\mathrm{c}_{i j}^{\lambda}=\beta_{i j}-h_{i j}$.

\section{Numerical illustration}

\subsection{Example}

Consider the non-linear transportation problem with 2 origins, 2 destination as follows:

- Transported cost $c_{i j}^{0}$

$$
c_{i j}^{0}=\left[\begin{array}{ll}
13 & 16 \\
10 & 12
\end{array}\right]
$$


- Shipped transportation cost $c_{i j}$

$$
c_{i j}=\left[\begin{array}{ll}
<5,13> & <6,11> \\
<4,13> & <2,13>
\end{array}\right]
$$

- Quality product matrix $q_{i j}$

$$
\left[q_{i j}\right]=\left[\begin{array}{ll}
0.8 & 0.5 \\
0.9 & 0.9
\end{array}\right]
$$

and

$$
\left[\gamma_{i j}\right]=\left[\begin{array}{ll}
10 & 10 \\
10 & 10
\end{array}\right]
$$

and the corresponding maximum cost of the transportation problem with costs $\beta_{i j}^{\prime}$ as $b=190$ (Tables $1-3)$.

Table 1. Input data

\begin{tabular}{|c|c|c|c|c|}
\hline Supply & Demand & Impurity & $\begin{array}{c}\text { Maximum impurity } \\
\text { received }\end{array}$ & Known location \\
\hline$a_{1}=6$ & $b_{1}=4$ & $f_{1}=1$ & $g_{1}=5$ & $(4,8)$ \\
$a_{2}=2$ & $b_{2}=4$ & $f_{2}=2$ & $g_{2}=8$ & $(7,9)$ \\
\hline
\end{tabular}

Table 2. For lower bound

\begin{tabular}{|l|l|l|l|}
\hline \multicolumn{2}{|c|}{ Distance } & \multicolumn{2}{c|}{ Unknown location } \\
\hline$d_{11}=1.33$ & $d_{12}=1.9$ & $x_{1}=5.38$ & $x_{2}=4.0$ \\
\hline$d_{21}=0$ & $d_{22}=3.0$ & $y_{1}=8.0$ & $y_{2}=9.1$ \\
\hline
\end{tabular}

Table 3. For upper bound

\begin{tabular}{|l|l|l|l|}
\hline \multicolumn{2}{|c|}{ Distance } & \multicolumn{2}{c|}{ Unknown location } \\
\hline$d_{11}=3.16$ & $d_{12}=0$ & $x_{1}=7.2$ & $x_{2}=5.3$ \\
\hline$d_{21}=1.65$ & $d_{22}=1.63$ & $y_{1}=9.0$ & $y_{2}=9.0$ \\
\hline
\end{tabular}

Hence, from equations (30), (31), the problem reduces to

$$
\max =\frac{190-13 w_{11}-15 w_{12}-10 w_{21}-12 w_{22}-6.5 y_{11}-11.4 y_{12}-6 y_{22}}{60+13 y_{11}+19 y_{12}+30 y_{22}}
$$

subject to 


$$
\begin{aligned}
& w_{11}+w_{12} \leq a_{1} \\
& w_{21}+w_{22} \leq a_{2} \\
& w_{11}+w_{21} \geq b_{1} \\
& w_{12}+w_{22} \geq b_{2} \\
& f_{1} w_{11}+f_{2} w_{21} \leq g_{1} \\
& f_{2} w_{12}+f_{2} w_{22} \leq g_{2}
\end{aligned}
$$

For $(i, j) \in E$, we have

$$
\lambda_{w}=\frac{\beta_{i j}-h_{i j}-\alpha_{i j}}{q_{i j}}
$$

so that (Table 4)

$$
h_{i j}=\beta_{i j}-\alpha_{i j}-q_{i j} \lambda_{w}
$$

Table 4 . Value of $h_{i j}$ and fuzzy cost corresponding to $\lambda$

\begin{tabular}{|l|l|l|l|}
\hline \multicolumn{2}{|c|}{ Value of $h_{i j}$} & \multicolumn{2}{c|}{ Fuzzy cost corresponding $\lambda$} \\
\hline$h_{11}=3.1$ & $h_{12}=0.1$ & $c_{11}^{0.49}=9.9$ & $c_{12}^{0.49}=10.9$ \\
\hline$h_{21}=4.1$ & $h_{22}=6.1$ & $c_{21}^{0.49}=8.9$ & $c_{22}^{0.49}=6.9$ \\
\hline
\end{tabular}

The optimal solution of problem (32) is obtained using the Kanti Swarup method (Tables 5, 6).

Table 5. Solution by the Kanti Swarup method

\begin{tabular}{|l|l|c|c|}
\hline \multicolumn{2}{|c|}{ Decision variable $w_{i j}$} & $\max \lambda$ & $z_{1}$ \\
\hline$w_{11}=3.23$ & $w_{12}=2.76$ & \multirow{2}{*}{0.49} & \multirow{2}{*}{91.46} \\
\hline$w_{21}=0.76$ & $w_{22}=1.23$ & & \\
\hline
\end{tabular}

Table 6. Solution by the fractional programming method

\begin{tabular}{|l|l|c|c|}
\hline \multicolumn{2}{|c|}{ Decision variable $w_{i j}$} & $\max \lambda$ & $z_{1}$ \\
\cline { 1 - 2 }$w_{11}=3.99$ & $w_{12}=2.01$ & \multirow{2}{*}{0.49} & \multirow{2}{*}{87.41} \\
\hline$w_{21}=0.02$ & $w_{22}=1.99$ & & \\
\hline
\end{tabular}

From the present model, it is seen that distances from origins to destinations play an important role in the transportation system. For simplicity, here we settled a $(2 \times 2)$ transportation problem, where the results reveal the following decisions: 
- The solution ( $\min Z=91.46)$ by the Kanti Swarup method is inferior to the solutions ( $\min Z=87.41)$ obtained by the fractional programming methods. But the allocations obtained by method 1 is more creditable (the allocation $W_{21}=0.02$ by fractional programming method is near about zero).

- Solutions obtained by this method occurred for more time complexity as well as space complexity, since, they are obtained in three steps, whereas for the fractional programming method, we obtain the results in a single step. So, the fractional programming method is a single-step method and the Kanti Swarup method is a multi-step method.

\section{Sensitivity analysis and its discussion}

For sensitivity, we consider the post-optimality condition that the transportation system does not affect the distances from sources to destinations. The corresponding results given below are obtained from the two methods discussed above (Tables 7, 8).

Table 7. Particular results by the Kanti Swarup method

\begin{tabular}{|l|l|c|c|}
\hline \multicolumn{2}{|c|}{ Decision variable $w_{i j}$} & $\max \lambda$ & $z_{1}$ \\
\cline { 1 - 2 }$w_{11}=2.03$ & $w_{12}=2.00$ & \multirow{2}{*}{0.49} & \multirow{2}{*}{71.03} \\
\hline$w_{21}=0.86$ & $w_{22}=1.30$ & & \\
\hline
\end{tabular}

Table 8. Particular results obtained by the fractional programming method

\begin{tabular}{|l|l|c|c|}
\hline \multicolumn{2}{|c|}{ Decision variable $w_{i j}$} & $\max \lambda$ & $z_{1}$ \\
\hline$w_{11}=3.0$ & $w_{12}=3.0$ & \multirow{2}{*}{0.49} & 6 \\
\cline { 1 - 2 }$w_{21}=1.0$ & $w_{22}=1.0$ & & \\
\hline
\end{tabular}

From the results obtained out of particular cases, that is by ignoring the measurement of distances of transportation, it is revealed that more criteria (here, distance) yield more total cost. So, in reality, if we ignore such matter, the results will be more profitable.

\section{Conclusions}

Transportation problem arises in many sectors. Industry, public society are shaped in this form. Here, for the first time, a non-linear transportation problem is proposed, where one part of the objective function is linear with usual transportation cost, and the second part is non-linear, due to the unit transportation cost which varies with distance from the source to the origin. We model a fuzzy transportation problem to overcome the uncertain nature of the real world. The model is defuzzified and optimised using the 
Kanti Swarup method and fractional programming methods. Here, one impurity constraint is considered to maintain the non-defective of the times. The method discussed will guide decision-makers in the field of logistics which are related to practical problems of life and also provide optimal solutions in a very simple and effective way. The technique to formulate an STP as a minimisation problem can be used to other types of transportation models or problems.

As the proposed model is one of the realistic models, so it can be formulated with other different types of uncertainty, like rough, fuzzy-rough, stochastic, fuzzy stochastic, etc. The problem also can be exchanged to a profit maximisation model instead of minimisation of the total cost. The problem can be solved with other types of soft computing methods, like a genetic algorithm, particle swarm optimisation, etc.

\section{Acknowledgement}

The authors would like to thank Barun Das and Prabhas Kumar Singh for their considerable help in editing the final version of this paper.

\section{References}

[1] Bit A.K., Biswal M.P., Alam S.S., Fuzzy programming approach to multi-objective solid transportation problem, Fuzzy Sets Syst., 1993, 57, 183-194.

[2] Bit A.K., Biswal M.P., Alam S.S., An additive fuzzy programming model for multi objective transportation problem, Fuzzy Sets Syst., 1993, 57, 313-319.

[3] BisWAL M.P., VERMA R., Fuzzy programming technique to solve a non-linear transportation problem, Fuzzy Math., 1999, 7, 723-730.

[4] Lin C.-J., Wen U.-P., A labeling algorithm for the fuzzy assignment problem, Fuzzy Sets Syst., 2004, $142,373-391$.

[5] Dutta D., Murthy Satyanarayana A., Fuzzy transportation problem with additional restriction, ARPN J. Eng. Appl. Sci., 2010, 5 (2), 36-40.

[6] Jimenez F., Verdegay J.L., Uncertain solid transportation problems, Fuzzy Sets Syst., 1998, 100, $45-57$.

[7] KANTI SwARUP, Linear fractional functional programming, Oper. Res., 1965, 12, 1029-1036.

[8] Singh P., SAXENA P.K., The multiobjective time transportation problem with additional restrictions, Eur. J. Oper. Res., 2003, 146, 460-476.

[9] Bellman R.R., ZADeH L.A., Decision making in a fuzzy environment, Manage. Sci., 1970, B17, 203-218.

[10] ZADEH L.A., Fuzzy sets, Inf. Contr., 1965, 8, 338-353.

[11] ZiMMERMANN H.J., Fuzzy programming and linear programming with several objective functions, Fuzzy Sets Syst., 1978, 1, 45-55.

[12] НітснСОск F.L., The distribution of a product from several sources to numerous localities, Studies in Appl. Math., 1941, 20 (1-4), 224-230.

[13] ApPA G.M., The Transportation problem and its variants, Oper. Res. Quart., 1973, 24 (1), 79-99.

[14] ANUKOKILA P., RADHAKRISHNAN B., ANJU A., Goal programming approach for solving multi-objective fractional transportation problem with fuzzy parameters, RAIRO Oper. Res., 2019, 53 (1), 157-178.

[15] KUMAR P.S., PSK method for solving mixed and type-4 intuitionistic fuzzy solid transportation problems, International J. Oper. Res. Inf. Syst., 2019, 10 (2), 20-53. 
[16] KUMAR P.S., PSK method for solving intuitionistic fuzzy solid transportation problems, Int. J. Fuzzy Syst. Appl., 2018, 7 (4), 62-99.

[17] KUMAR P.S., A note on a new approach for solving intuitionistic fuzzy transportation problem of type-2, Int. J. Log. Syst. Manage., 2018, 29 (1), 102-129.

[18] KUMAR P.S., HUSSAIN R.J., A systematic approach for solving mixed intuitionistic fuzzy transportation problems, Int. J. Pure Appl. Math., 2014, 92 (2), 181-190.

[19] Kumar P.S., Intuitionistic fuzzy solid assignment problems: a software-based approach, Int. J. Syst. Assur. Eng. Manage., 2018, 10 (4), 661-675.

[20] Kumar P.S., A simple method for solving type-2 and type-4 fuzzy transportation problems, Int. J. Fuzzy Logic Intel. Syst., 2016, 16 (4), 225-237.

[21] LIANG T.F., Interactive multi-objective transportation planning decisions using fuzzy, linear programming, Asia-Pacific J. Oper. Res., 2008, 25 (1), 11-31.

[22] VeRMA R., BISWAL M.P., BISWAS A., Fuzzy programming technique to solve multi objective transportation problems with some non-linear membership functions, Fuzzy Sets Syst., 1997, 91, 37-43.

[23] LI L., LAI K.K., A fuzzy approach to the multi-objective transportation problem, Comp. Oper. Res., 2000, 27, 43-57.

[24] SHELl E., Distribution of a product by several properties, Directorate of Management Analysis, Proc. Second Symposium in Linear Programming, 1955, 2, 615-642.

[25] RAMAKRISHNAN C.S., An improvement to Goyal's modified VAM for the unbalanced transportation problem, J. Oper. Res. Soc., 1988, 39 (6), 609-610.

[26] ShafaAt A., Goyal S.K., Resolution of degeneracy in transportation problems, J. Oper. Res. Soc., 1988, 39 (4), 411-413.

[27] ARShAm H., KAHN A.B., A simplex-type algorithm for general transportation problems. An alternative to stepping-stone, J. Oper Res. Soc., 1989, 40 (6), 581-590.

[28] Gass S.I., On solving the transportation problem, J. Oper. Res. Soc., 1990, 41 (4), 291-307.

[29] ADLAKHA V., KowALSKI K., A simple heuristic for solving small fixed-charge transportation problems, Omega, 2003, 31 (3), 205-211.

[30] BARR R.S., Glover F., KLIngman D., A new optimization method for large scale fixed charge transportation problems, Oper. Res., 1981, 29 (3), 448-463.

[31] HALEY K.B., New methods in mathematical programming - the solid transportation problem, Oper. Res., 1962, 10 (4), 448-463. 